\title{
Supratentorial Embryonal Tumors in the Elderly: Diagnostic Pitfalls and Clinical Prognosis
}

\author{
Pierluigi GRANAROLI ${ }^{1}$, Federica NOVEGNO ${ }^{1}$, Tommaso LUNARDI ${ }^{2}$, Pierpaolo LUNARDI ${ }^{1}$ \\ ${ }^{1}$ Policlinico Tor Vergata, Medical School, Department of Neurosurgery, Rome, Italy \\ ${ }^{2}$ Ospedale San Paolo, Department of Radiology, Rome, Italy
}

Corresponding author: Federica NOVEGNO federicanovegno@hotmail.it

\section{ABSTRACT}

Supratentorial embryonal tumors are very rare malignant tumors of neuroectodermal origin, characterized by an aggressive clinical behavior. They occur prevalently in children. They have been sporadically described in adults and represent an even rarer occurrence in elderly patients, raising many issues on the diagnostic pitfalls and their appropriate management. We present an unusual case of embryonal tumor in a 62-year-old man who presented with speech disorder, and partial deficit of the left $3^{\text {rd }}$ and $5^{\text {th }}$ cranial nerves secondary to a left temporo-insular embryonal tumor: the clinico-radiological features, histopathological insights, therapeutic options and results are discussed along with a review of the most relevant literature, addressing the specific issue of differential diagnosis and the expected results in the elderly population.

KEYWORDS: Elderly, Embryonal tumor, Prognosis, Supratentorial

\section{INTRODUCTION}

$\mathrm{T}$ The embryonal tumors other than medulloblastoma represent a heterogeneous group of malignancies characterized by poorly differentiated neuroepithelial cells and generally aggressive behavior, localized in the supratentorial compartment. They have been historically indicated as supratentorial primitive neuroectodermal tumor (sPNET) (6). These tumors occur prevalently in children, representing about $2.8 \%$ of all primary brain tumors of childhood and adolescence. They represent an extremely rare occurrence in elderly patients aged 60 years or older, raising many issues on the diagnostic pitfalls and management strategy $(1,3,4,5,7,10,12,13,15)$.

We report the case of a 62-year-old man affected by a temporo-insular embryonal tumor with a review of the most relevant literature, addressing the specific issue of differential diagnosis and the expected results in the elderly population.

\section{CASE REPORT}

A 62-year-old male patient presented to our Department with a two-month history of short-term memory impairment, associated with aphasia nominum, semantic paraphasias, numbness sensation of the left hemi-face and partial left third cranial nerve deficit.

Magnetic resonance imaging (MRI) of the brain showed the presence of a left temporo-insular lesion (Figure 1A-F). The patient underwent subtotal removal through a left frontotemporal craniotomy. The lesion was classified as SPNET, World Health Organization (WHO) grade IV (Figure 2A-F).

The postoperative course was characterized by a slight improvement of the preoperative symptoms. He underwent chemotherapy and radiotherapy. A 6 month-postoperative $\mathrm{MRI}$ documented tumoral re-growth, for which a reoperation was proposed. However, the patient refused. In the next four months, he experienced a progressive sensory deterioration, until a state of coma and subsequent death.
Pierluigi GRANAROLI (1) : 0000-0002-5814-9997 Federica NOVEGNO (D): 0000-0001-7635-9907
Tommaso LUNARDI (10 : 0000-0001-6881-4383

Pierpaolo LUNARDI (D) : 0000-0003-0812-9805 


\section{DISCUSSION}

The term supratentorial PNET was originally used to describe central nervous system (CNS) tumors that histologically resembled medulloblastoma, but originated outside the cerebellum $(9,12)$. The recent WHO classification of CNS tumors, published in 2016 (6), introduced major changes regarding these tumors' definition, starting with the removal of the term PNET. Much of the re-classification was driven by the recognition that many of these rare tumors display amplification of the C19MC region on chromosome 19 (19q13.42). Therefore, it includes embryonal tumors with abundant neuropil and true rosettes (ETANTR), ependymoblastoma and medulloepithelioma. Recent studies have shown that adult SPNET cases present some significant differences: high incidence of TP53 mutations, no amplification of the c-myc/N-myc genes, and presence of IDH1 mutations (2).

The incidence of cases reported in the adult population is extremely low, accounting for less than $0.5 \%$; they occur prevalently in the third decade of life, resembling the presence of two peaks in the age profile for SPNET onset: childhood and middle age $(5,15)$. During the last decade, the interest on adult patients affected by SPNET has increased progressively due
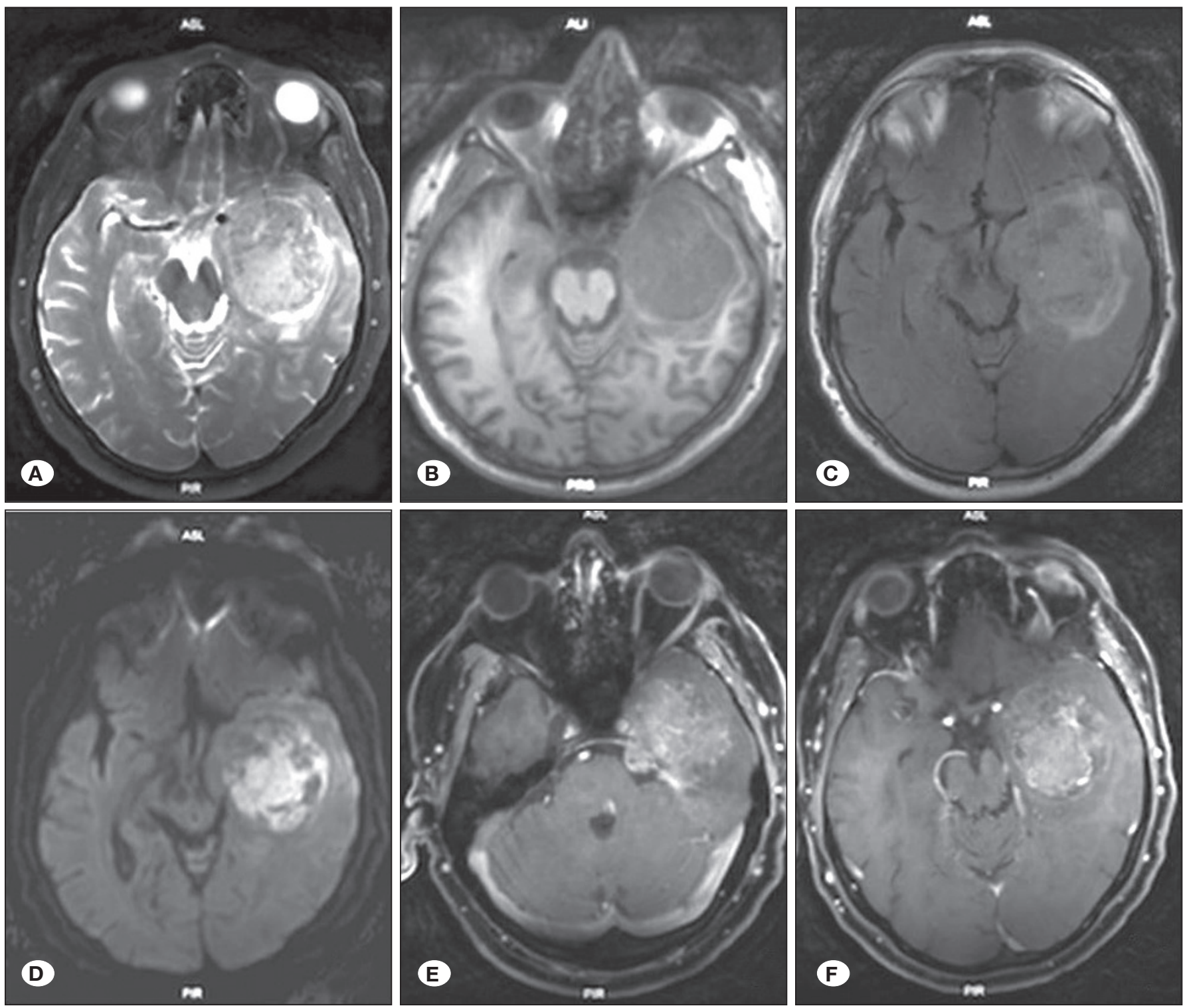

Figure 1: MRI showing a huge well circumscribed, oval shaped, left temporo-insular lesion (about $5 \mathrm{~cm}$ diameter), associated with perilesional edema; an extraaxial origin from the anterior edge of the left tentorium with a small infratentorial component invading the homolateral pre-pontine cistern was depicted. A-C) T2- and B) T1-weighted axial images. The tumor appeared isointense to cortex lesion on A) T2-weighted and C) T2 - FLAIR MRI images, and restricted in D) diffusion sequences (DWI). E-F) On T1-weighted axial images with gadolinium there was inhomogeneous contrast enhancement with peripheral colliquative areas, involving the entire left Meckel cave, deforming the third and the left lateral ventricle and displacing the ipsilateral middle cerebral artery (MCA). These findings were highly suspicious for a lesion of extra-axial origin, like a meningioma. 
to the better identification of these tumors $(5,15)$. According to the literature review, there are only 20 cases of patients aged 60 years or older reported so far, who deserve a particular discussion (Table I).

Patients ranged in age from 60 to 88 years (mean 68.6 years) with a male predominance (ratio 2.5:1). In these cases, most of the lesions were located in the temporal and parietal lobes, compared with a prevalent localization in the frontal lobe in adult patients (15); one patient had a corpus callosum seeding, whereas bilateral ventricles lesions were observed in another one. None of them had a pineal localization, as reported in the general adult series (15).

The most common presenting symptoms were focal neurological deficits and intracranial hypertension-related symptoms; seizures, frequently occurring in children, were not reported.

On neuroradiological examination, sPNETs appeared as large masses, rarely associated with necrosis cysts, calcification and hemorrhage (11). They displayed heterogeneous signal intensity, with the solid portion of the tumor typically showing hypointensity on T1-weighted images and hyperintensity on T2-weighted images; intense homogenous enhancement was often described on contrasted T1-weighted sequences. Peritumoral edema was also depicted $(5,11)$. On the basis of these findings, the rarity of this tumor in the elderly may raise some difficulties in the differential diagnosis. SPNET in adults usually looks like a high-grade astrocytoma or anaplastic oligodendroglioma on computed tomography and $\mathrm{MRI}$; however tumor cyst, calcification and lobulating contour with good demarcation may be more frequently observed in SPNET than in high-grade gliomas. It may appear more similar to a meningioma as in our case. This hypothesis is sustained by epidemiological data (meningiomas are common intracranial neoplasms with a maximum incidence between the fifth and sixth decade), presence of degenerative necrotic - cyst/pseudocyst, perilesional edema (depicted in 60\% of meningiomas) and intense and homogeneous contrast enhancement with a "dural tail" sign associated $(5,8,14)$.

The initial staging should include spinal MRI and cerebrospinal fluid (CSF) cytology, in order to plan cranio-spinal radiotherapy or intrathecal chemotherapy, as generally applied in children. Indeed, CSF spreading is one of the few prognostic factors correlated with outcome in adult sPNET (15).

Age was the other factor associated with worse survival. According to Zheng et al.(15), the 1 year survival rate is better for patients aged $<60$ years ( $83.3 \%$ versus $40 \%$ ), although not statistically significant.

Generally speaking, sPNET in adults are rarer and usually have a worse prognosis compared to their infratentorial counterpart (medulloblastomas), as well as compared with sPNET in the pediatric population. More than 2/3 of the entire series of elderly patients died after a mean follow-up of 10.9 months (range 2-62 months) $(5,13,15)$. Due to the small number of cases analysed, we are not able to draw conclusions, especially with regard to those patients who had a better clinical follow-up; however, data emerging from the literature depicted age at onset, surgical resection, extent of resection and radiotherapy as good prognostic factors (15). Zheng et al. observed that radiotherapy and both chemotherapy and
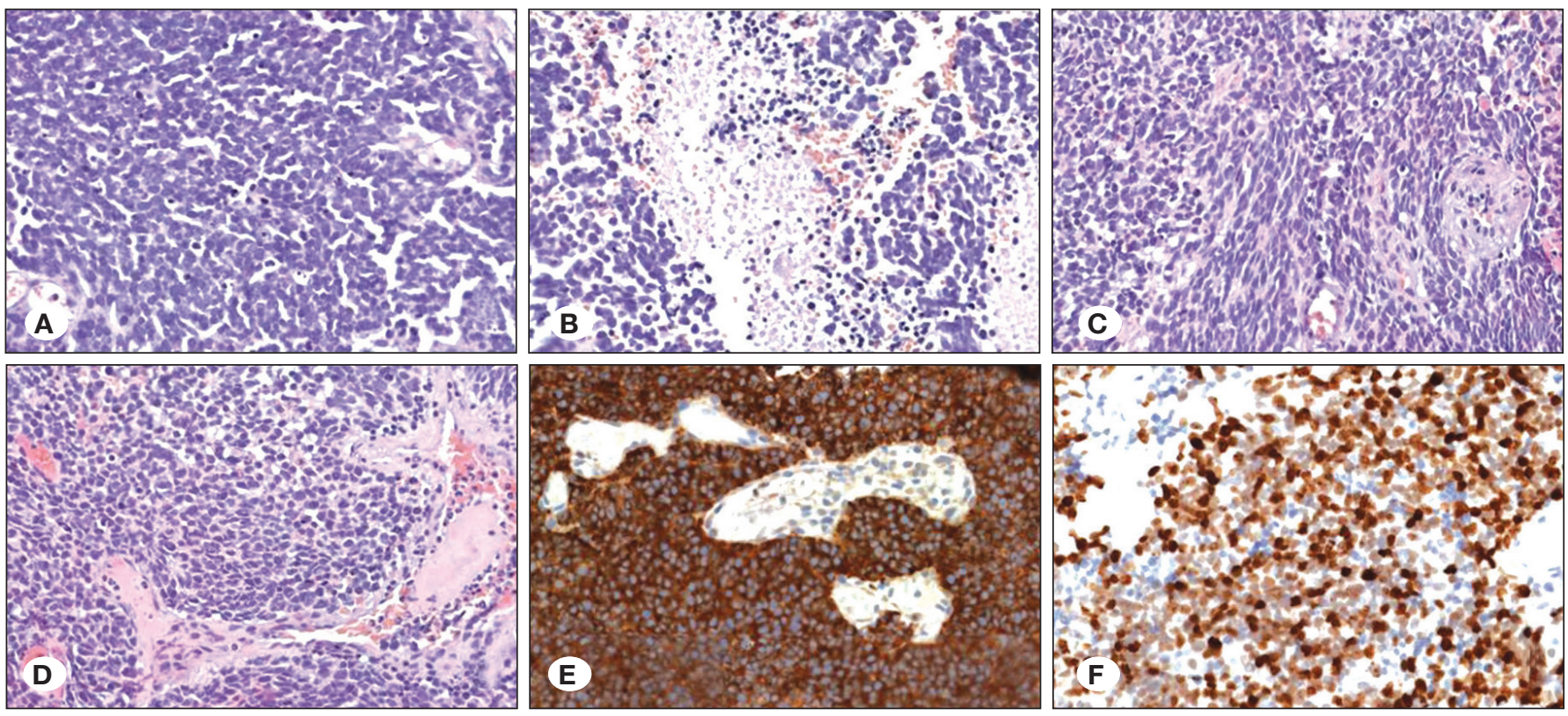

Figure 2: A) Hematoxylin \& Eosin (H\&E) stain (400x): small cell monomorphic proliferation with addensed chromatin, little cytoplasm, with some thickening of the blood vessel wall, B) H\&E stain (400x): presence of oat-cells (short, bluntly spindle-shaped, anaplastic cell), conforming to PNETs, C) H\&E stain (200x): vital cell populations with pathways of coagulative necrosis, D) H\&E stain (400x): presence of mitotic cells and apoptotic bodies (dense and small nucleus). This feature represents the high grade malignancy, E) (400x): Immunohistochemistry of synaptophysin, bright diffuse staining throughout the entire cytoplasm of all cells, F) (400x): Ki67 antigen immunostaining (MIB 1 monoclonal antibody): mark in proliferating cells (G1-S e M phases), nuclear positivity $>85-90 \%$ 


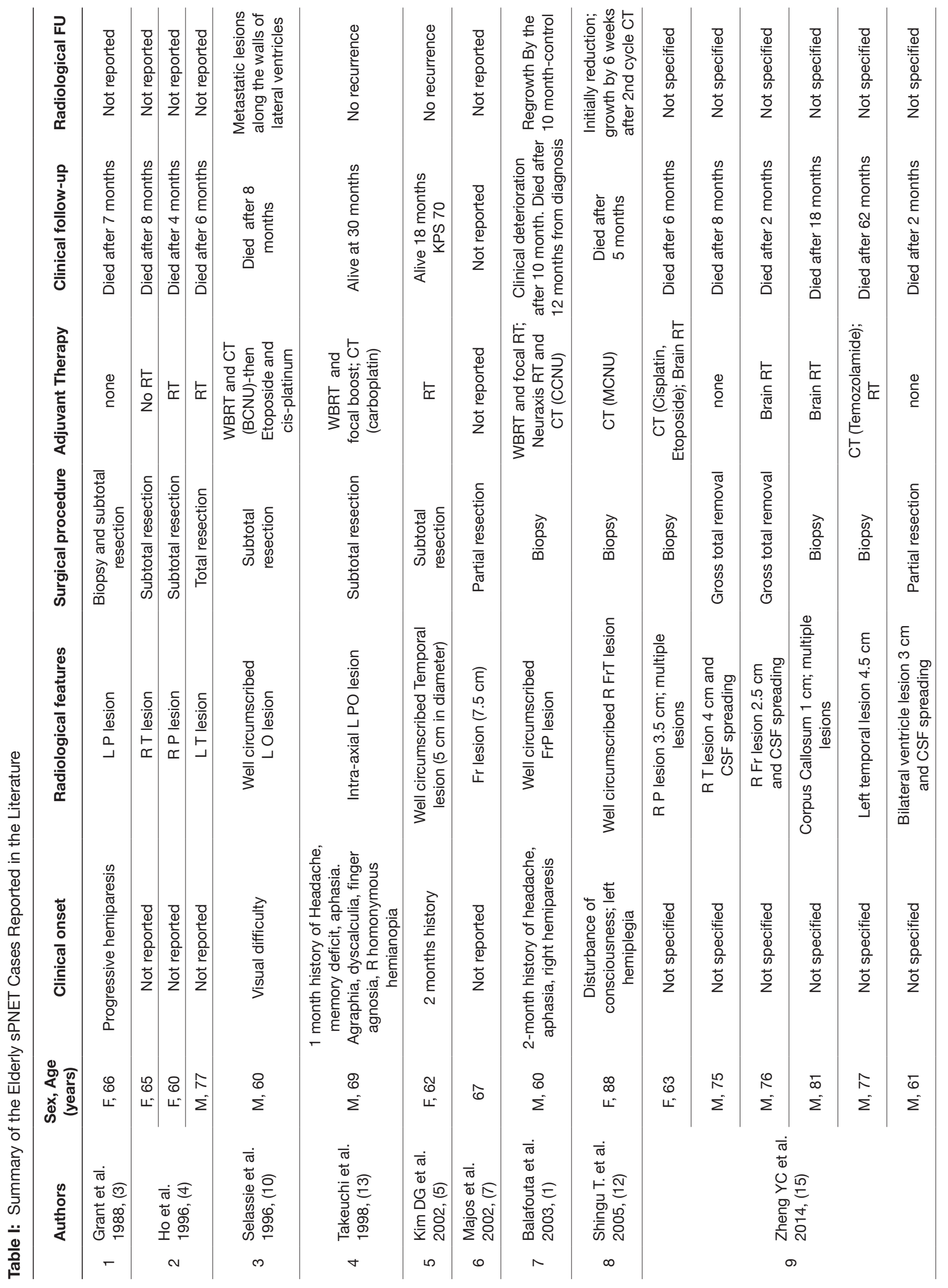


radiotherapy were marginally significant for better survival, whereas chemotherapy alone did not improve survival at all (15). However, due to a relative radioresistance of these tumors compared to medulloblastoma, the addition of multiregimen chemotherapy is considered of therapeutic value.

\section{CONCLUSION}

There is a general agreement to consider complete removal of the tumor when feasible; followed by adjuvant radiotherapy and chemotherapy, even in elderly patients should the general conditions allow the entire treatment.

\section{REFERENCES}

1. Balafouta MJ, Kouvaris JR, Miliaddu AC, Papacharalampous $\mathrm{XN}$, Kolokouris DV, Kouloulias VE, Mariolis AD, Vlahos LJ: Primitive neuroectodermal tumor in a 60-year-old man: A report and literature review. Br J Radiol 76:62-65, 2003

2. Gessi M, Setty P, Bisceglia M, Zur Muehlen A, Lauriola L, Waha A, Giangaspero F, Pietsch T: Supratentorial primitive neuroectodermal tumors of the central nervous system in adults: Molecular and histopathologic analysis of 12 cases. Am J Surg Pathol 35(4):573-582, 2011

3. Grant JW, Steart PV, Gallagher PJ: Primitive neuroectodermal tumors of the cerebrum: A histological and immunohistochemical study of 10 cases. Clin Neuropathol 7(5):228-233, 1988

4. Ho YS, Hsieh LL, Chen JS, Chang CN, Lee ST, Chiu LL, Chin TY, Cheng S: p53 gene mutation in cerebral primitive neuroectodermal tumor in Taiwan. Cancer Lett 104:103-113, 1996

5. Kim DG, Lee DY, Paek SH, Chi JG, Choe G, Jung HW: Supratentorial primitive neuroectodermal tumors in adults. J Neurooncol 60:43-52, 2002

6. Louis DN, Perry A, Reifenberger G, von Deimling A, FigarellaBranger D, Cavenee WK, Ohgaki H, Wiestler OD, Kleihues P, Ellison DW: The 2016 World Health Organization Classification of Tumors of the Central Nervous System: A summary. Acta Neuropathol 131(6):803-820, 2016

7. Majos C, Alonso J, Aguilera C, Serralonga M, Acebes JJ, Arus C, Gili J: Adult primitive neuroectodermal tumor: Proton MR spectroscopic findings with possible application for differential diagnoses. Radiology 225:556-566, 2002

8. Pickuth $\mathrm{D}$, Leutloff U: Computed tomography and magnetic resonance imaging findings in primitive neuroectodermal tumours in adults. Br J Radiol 69(817):1-5, 1996

9. Rorke LB, Hart MN, Mclendon RE: Supratentorial primitive neuroectodermal tumor (PNET). In: Kleihuse P, Cavanee WK (eds). Pathology and Genetics of Tumours of the Nervous System. Lyon: YARC Press, 2000:141-144

10. Selassie L, Rigotti R, Kepes JJ, Towfighi J: Adipose tissue and smooth muscle in a primitive neuroectodermal tumor of cerebrum. Acta Neuropathol (Berl) 87:217-222, 1994

11. Shi H, Kong $X, X u H, X u$ L, Liu D: MRI features of intracranial primitive neuroectodermal tumors in adults: Comparing with histopathological findings. J Huazhong Univ Sci Technolog Med Sci 24(1):99-102, 2004 
12. Shingu T, Kagawa T, Kimura Y, Takada D, Moritake K, Hoshii Y: Supratentorial primitive neuroectodermal tumor in an aged patient-case report. Neurol Med Chir 45(10):530-535, 2005

13. Takeuchi H, Kabuto M, Sato K, Kubota T: Cerebral primitive neuroectodermal tumor in an elderly patient with long-term survival. J Clin Neurosci 5:466-469, 1998
14. Wiemels J, Wrensch M, Claus EB: Epidemiology and etiology of meningioma. J Neurooncol 99(3):307-314, 2010

15. Zheng YC, Jung SM, Lee ST, Chang CN, Wei KC, Hsu YH, Wu CT, Liao CC, Lin CL, Lu YJ, Huang YC: Adult supratentorial extra-pineal primitive neuro-ectodermal tumors. J Clin Neurosci 21(5):803-809, 2014 